\title{
A REACTIVE OBSTACLE AVOIDANCE SYSTEM FOR AN AUTONOMOUS UNDERWATER VEHICLE
}

\author{
Mike Eichhorn \\ Institute of Automation and Systems Engineering \\ Technical University of Ilmenau, PO Box 100565, 98684 Ilmenau, Germany \\ (phone +493677 691421; fax +493677691434 \\ e-mail: Mike.Eichhorn@TU-Ilmenau.de)
}

\begin{abstract}
This paper presents a reactive/local level of an Obstacle Avoidance System for an Autonomous Underwater Vehicle (AUV). The specific requirements of the underwater world, the computational capacity and the sensors of the vehicle as well as its manoeuvrability were considered by the choice and the development of strategies used. Such requirements include the sea current information, the consideration of moving objects and the constrained view of sonar. Copyright (C) 2005 IFAC
\end{abstract}

Keywords: Autonomous vehicle, Obstacle avoidance, Autonomous Underwater Vehicle

\section{INTRODUCTION}

This article presents a part (guidance system in special situations) of the current research project "DeepC". On this project an interdisciplinary consortium, consisting of German companies and research establishments, works under guidance of the Federal Ministry for research and development on the development of an AUV (DeepC, 2004), (BMBF, 2001). This underwater vehicle should be able to accomplish a given mission and act independently in unexpected situations (emerging obstacles, loss of components). The vehicle is specified by the following performance parameters:

Table 1 Vehicle Parameters

\section{Parameter}

Value

Limits of the vehicle L 5,80 m B 2,30 $\mathrm{m} \mathrm{H} \mathrm{1,70} \mathrm{m}$

Weight

Diving Depth

$4000 \mathrm{~m}$

Cruise/Max speed

$4 \mathrm{kts} / 6 \mathrm{kts}$

Endurance

$40 \mathrm{~h}$

Weight Payload

$300 \mathrm{~kg}$

Energy
A schematic figure of the vehicle, including with its most important drive and manoeuvring equipment is represented in Fig. 1. DeepC obtains here good positioning and manoeuvring qualities in standing position and at less speed by the thrusters (3). At higher speeds, the manoeuvring of the vehicle in done by a combination of the thrusters (3), the fin units (4) and rotation speed differences of the main drives (5).

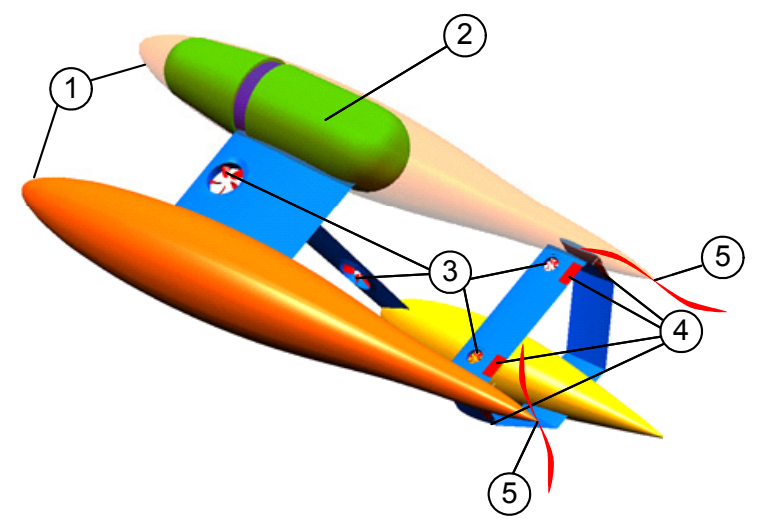

Fig. 1. Structure of the AUV DeepC 


\section{REACTIVE EVASIVE CONTROL BY MEANS OF GRADIENT LINES}

The reactive evasive control is a part of the obstacle avoidance system of the AUV DeepC. Detailed information about this system are presented in Eichhorn (2004).

The idea of the method presented in this chapter is based on the generation of lines, which point to the goal from any position of the area of operation. The target course of the vehicle is determined by calculation of the course gradient $(\mathbf{G})$ at the current vehicle position. In this way the vehicle can be led past the obstacles to the goal from its current position. Since in the reactive level only the $x-y$ plane of the obstacles is considered, only the 2Dworld is to be treated in this section. However, by appropriate modifications it is also possible to use this procedure for the 3D-World.

The method presented in this chapter is based on the work of Guldner $(1995,1997)$. Here gradient lines are produced by artificial harmonious dipole potentials. The negative characteristics of the artificial potential fields can be compensated by the use of such dipole potentials. Such characteristics include the trapping of the vehicle in local minima, oscillating movements in narrow passages as well as closing of other possible routes by the resulting repulsive field at the entrance of a passage.

In this paper a new method is presented, which makes the production of the gradients from a geometrical construction of the gradient lines possible. Furthermore, possibilities of avoiding obstacles using the speed information of the seacurrent and the obstacles are pointed out.

\subsection{Production of gradient lines by harmonious dipole potentials}

For the following variants it is assumed that a circle describes an obstacle.

If one takes the circle as a positive point charge and the goal as a negative point charge, then this structure forms a dipole and its lines of flux lead from the positive to the negative charge. The value for the negative charge in the goal is selected to -1 . The positive charge of the obstacle can be expressed by the equation:

$$
q=\frac{R}{R+D}
$$

$R$ is the radius of the obstacle circle. $D$ describes the distance between the two charges. By equation (1) it is guaranteed that all gradient lines, which begin within the circle, are led out to end in the goal. Gradient lines, which begin outside of the circle, run exclusive outside of the circle. For the proof, it can be referred to Guldner $(1995,1997)$.

For the determination of the resulting gradient $\mathbf{G}$ at the current vehicle position, the two gradients for the positive and negative charges are to be computed separately and summarized by superposition.

Fig. 2 shows the potential field with its gradient lines, developed in the way described previously.

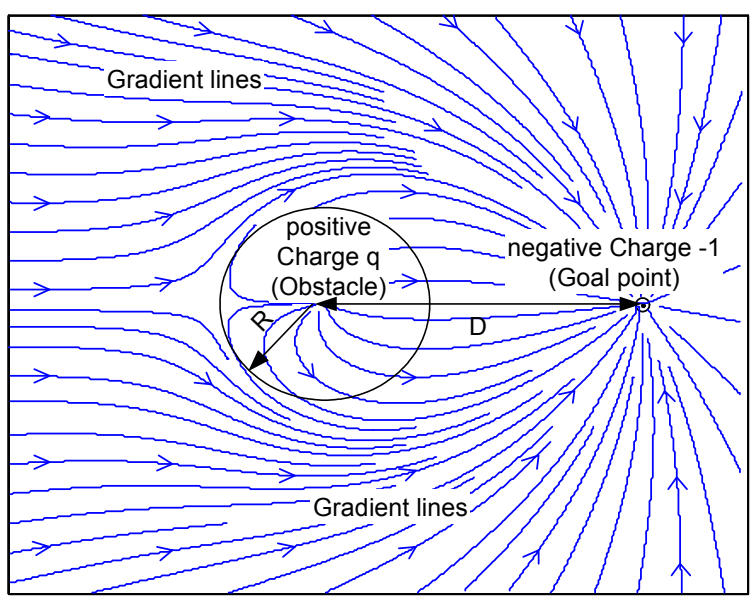

Fig. 2. Gradient lines of the dipole

Since the gradient lines can tangents the obstacle (however, not cut it), the radius $R$ of the circle must be increased by an amount, which prevents a collision between vehicle and object. Here an increase by n-multiples of the vehicle length is appropriate. The circle developed in this way is also called the safety zone (Guldner, 1997).

If the vehicle is on the side of the goal and drives in the proximity of the symmetry axis of the dipole it is forced to go around the obstacle before reaching the obstacle (see Fig. 2). Strong guidance commands are necessary, in order to keep the vehicle on the given gradient line. Furthermore, the form of the gradient lines within this range and in the laterals does not correspond to an optimal travel route. These effects increase with the increase in the parameters $R$ and $D$. These disadvantages are to be eliminated in the next section by the geometrical construction of gradient lines for optimal guidance.

\subsection{Production of gradient lines by geometrical construction}

To start with, it is mentioned here that the gradient lines produced in this section do not have physical "models". They must only lead to an optimal travel route.

To accomplish this, the area of operation is divided into individual sectors (see Fig. 3). The division into sectors depends on the vehicle position $\mathbf{x}_{\mathrm{veh}}$, the obstacle center $\mathbf{x}_{\mathbf{0 b s t}}$ and the goal position.

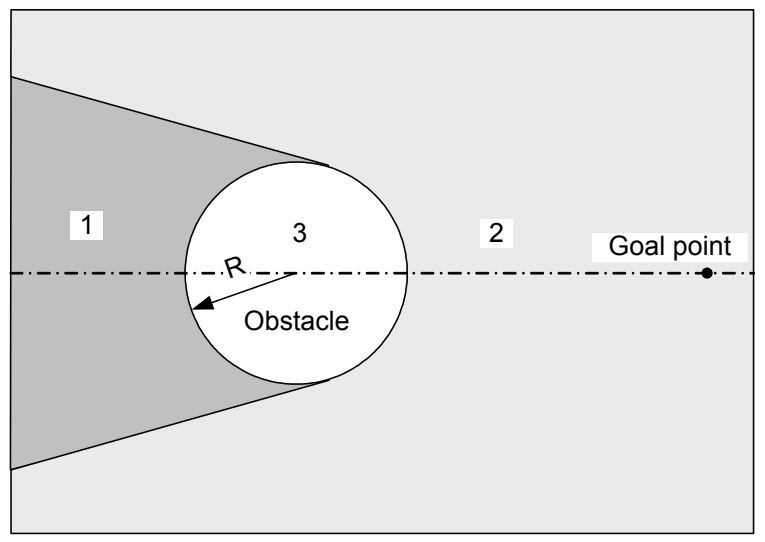

Fig. 3. Division of the area of operation into sectors 
If the condition $\left(\left|\mathbf{x}_{\mathbf{v e h}}-\mathbf{x}_{\text {obst }}\right|<R\right)$ is fulfilled, the vehicle is located inside the safety circle. Here sector 3 is active. The gradient can then be expressed by the following equation:

$$
\begin{aligned}
& \mathbf{x}_{\text {obst }}^{\text {dist }}=\mathbf{x}_{\text {veh }}-\mathbf{x}_{\text {obst }} \\
& \mathbf{G}=\frac{\mathbf{x}_{\text {obst }}^{\text {dist }}}{\left|\mathbf{x}_{\text {obst }}^{\text {dist }}\right|} .
\end{aligned}
$$

The activation of the sector 3 seems impossible in the first moment. Here it is however said again that the radius $R$ describes the security zone, in which the obstacle lies. Therefore, it is quite possible that a vehicle can penetrate this safety zone (because of strong current or late detection of the obstacle). In such a case the vehicle must be led away from the circle, which requires a description of a gradient inside the circle.

If the circle covers the view between vehicle and goal, there is no direct way to reach the goal (sector 1). Here the direction vector of the tangent from $\mathbf{x}_{\mathbf{v e h}}$ to the circle must be determined in dependence of the orientation of $\mathbf{x}_{\mathbf{v} \text { eh }}$ to the symmetry axis. The gradient is thereby the unit direction vector $\mathbf{x}_{\text {tangent }}^{\text {direction }}$ :

$$
\mathbf{G}=\frac{\mathbf{x}_{\text {tangent }}^{\text {direction }}}{\left|\mathbf{x}_{\text {tangent }}^{\text {direction }}\right|}
$$

If there is a direct connection between vehicle and goal, then sector 2 is active.

$$
\begin{aligned}
& \mathbf{x}_{\text {goal }}^{\text {dist }}=\mathbf{x}_{\text {veh }}-\mathbf{x}_{\text {goal }} \\
& \mathbf{G}=\frac{\mathbf{x}_{\text {goal }}^{\text {dist }}}{\left|\mathbf{x}_{\text {goal }}^{\text {dist }}\right|}
\end{aligned}
$$

In order to achieve a continuous transition between the gradient lines from the safety zone to the gradient lines of the sectors 1 and 2 , a radius $R_{\text {slid }}$ is introduced. In the resulting ring between $\mathrm{R}$ and $R_{\text {slid, }}$, a weighted superposition using a weighting factor $\alpha$ between the gradients outside and within the circle is done according to the following relation:

$$
\begin{aligned}
\alpha & =\frac{\left|\mathbf{x}_{\text {veh }}-\mathbf{x}_{\text {obst }}\right|-R}{R_{\text {slid }}-R} \\
\mathbf{G} & =\alpha \underbrace{\mathbf{G}_{\text {sector10r2 }}}_{\begin{array}{c}
\text { outside of } \\
\text { circle }
\end{array}}+(1-\alpha) \underbrace{\mathbf{G}_{\text {sector3 }}}_{\begin{array}{c}
\text { inside of } \\
\text { circle }
\end{array}}
\end{aligned}
$$

Fig.4 shows a gradient field produced in this way.

\subsection{Inclusion of speed information}

Under real environmental conditions it can occur that an obstacle moves (described through $\mathbf{v}_{\text {obst }}$ ) or a strong sea-current $\mathbf{v}_{\text {current }}$ is present. In such cases it would be meaningful to let these speed information also flow into the generation of the gradient lines.

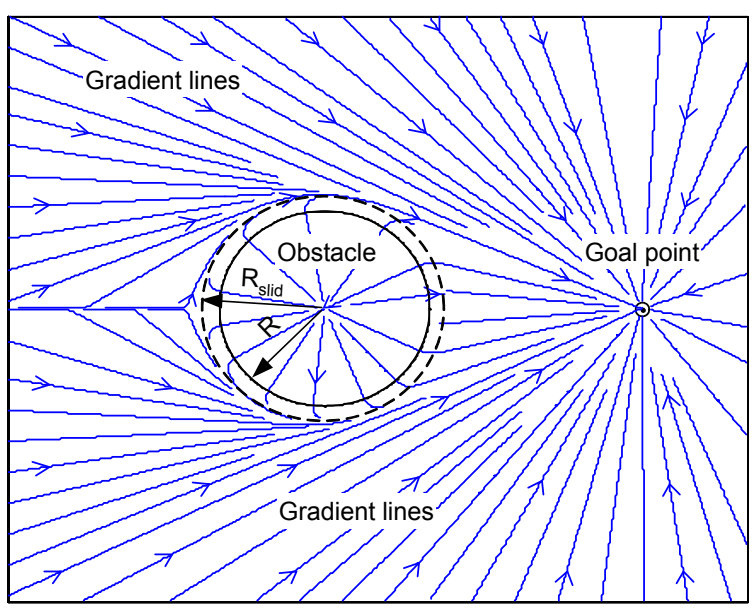

Fig.4. Gradient lines by geometrical construction

Therefore, it would be possible to begin an evasive manoeuvre earlier if an obstacle moves towards the vehicle or a strong back current is present. A possibility to accomplish this, would be the enlargement of the safety zone toward a resulting speed $\mathbf{v}_{\text {res }}$, which is described by the following relation:

$$
\mathbf{v}_{\text {res }}=\mathbf{v}_{\text {obst }}-\mathbf{v}_{\text {current }}
$$

The safety zone is transferred from a circle form with the radius $R$ into an ellipse (see Fig.5). The geometrical dimensions of the resulting ellipse are determined by the following equations.

$$
\begin{aligned}
& a_{\text {ellipse }}=R\left(1+v_{\text {rel }}\right) \\
& b_{\text {ellipse }}=R \sqrt{\left(1+v_{\text {rel }}\right)} \\
& \theta_{\text {ellipse }}=\tan ^{-1}\left(\frac{v_{\text {res }}^{y}}{v_{\text {res }}^{x}}\right)
\end{aligned}
$$

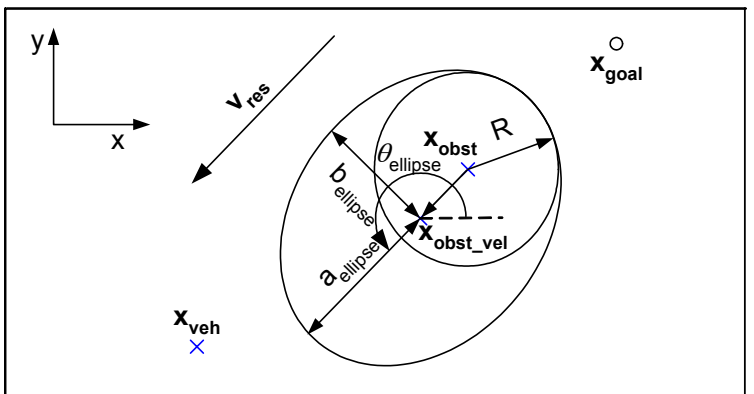

Fig.5. Deformation of the safety zone

The coefficient $v_{r e l}$ describes the relationship between the amount of the resulting speed $\left|\mathbf{v}_{\text {res }}\right|$ and the given cruising speed $v_{\text {cruise }}$ of the vehicle.

$$
v_{\text {rel }}=\frac{\left|\mathbf{v}_{\text {res }}\right|}{v_{\text {cruise }}}
$$

The centre of the ellipse can be expressed as follows:

$$
\mathbf{x}_{\text {obst_vel }}=\mathbf{x}_{\text {obst }}+\left[\begin{array}{l}
\cos \theta_{\text {ellipse }} R v_{\text {rel }} \\
\sin \theta_{\text {ellipse }} R v_{\text {rel }}
\end{array}\right]
$$




\subsection{Application to elliptical safety zones}

In the preceding descriptions it was assumed that a circle describes an obstacle. Since a circle describes many obstacles unfavourably, an elliptical form for such obstacles is more suitable. The adjustment of the presented method to an elliptical form would need much computational effort and would not be feasible at all. Here a pre-transformation must be accomplished, which transfers a turned ellipse with the position $\mathbf{x}_{\text {ellipse }}^{\text {center }}$ into a circle lying in the origin. The computation of the gradient lines is then done for circular obstacles, as described in section 2.2 and then an inverse transformation follows. The transformation for any point $\mathbf{x}$ in this space consists of several steps (see Fig. 6):

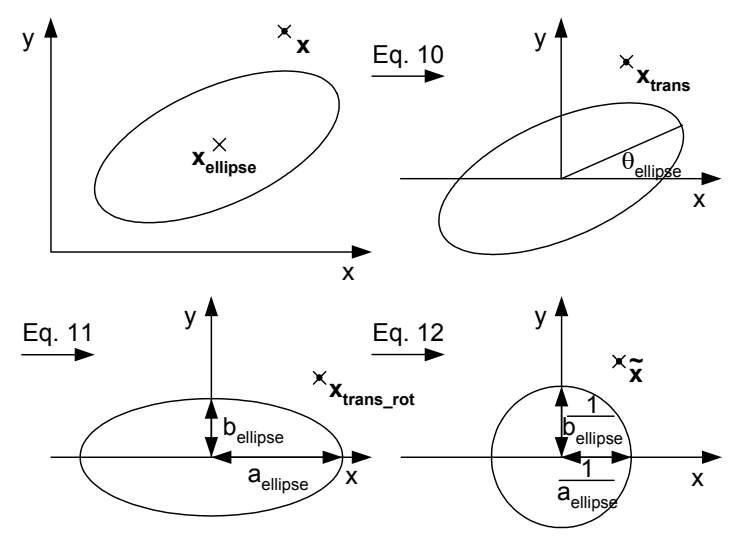

Fig. 6. Steps of the transformation

1) Translation of the point by $\mathbf{x}_{\text {ellipse }}^{\text {center }}$ (Shift of the ellipse into the origin of the display space.):

$$
\mathbf{x}_{\text {trans }}=\mathbf{x}-\mathbf{x}_{\text {ellipse }}^{\text {center }}
$$

2) Rotation of the point around the rotation angle of the ellipse $\theta_{\text {ellipse }}$ (Rotation of the ellipse to orient axis-parallel to the ellipse in the display space.):

$$
\mathbf{x}_{\text {trans_rot }}=\left[\begin{array}{cc}
\cos \theta_{\text {ellipse }} & \sin \theta_{\text {ellipse }} \\
-\sin \theta_{\text {ellipse }} & \cos \theta_{\text {ellipse }}
\end{array}\right] \mathbf{x}_{\text {trans }}
$$

3) Scaling of the point with the inverse values of the semi axis and minor axis ( $a_{\text {ellipse }}$ and $b_{\text {ellipse }}$ ) of the ellipse (Accomplishment of a circle for the ellipse with $R=1$.):

$$
\tilde{\mathbf{x}}=\left[\begin{array}{cc}
\frac{1}{a_{\text {ellipse }}} & 0 \\
0 & \frac{1}{b_{\text {ellipse }}}
\end{array}\right] \mathbf{x}_{\text {trans_rot }}
$$

After execution of this transformation the actual computations can be accomplished in the display space. The gradient $\mathbf{G}$ computed here must be back transformed again from the display space into the original space (equation (13), (14)).
Rescaling:

$$
\mathbf{G}_{\text {rescal }}=\left[\begin{array}{cc}
a_{\text {ellipse }} & 0 \\
0 & b_{\text {ellipse }}
\end{array}\right] \tilde{\mathbf{G}}
$$

Re-rotation:

$$
\mathbf{G}=\left[\begin{array}{cc}
\cos \theta_{\text {ellipse }} & -\sin \theta_{\text {ellipse }} \\
\sin \theta_{\text {ellipse }} & \cos \theta_{\text {ellipse }}
\end{array}\right] \mathbf{G}_{\text {rescal }}
$$

\subsection{Application to many obstacles}

In the previous descriptions, only an individual obstacle in the area of operation was regarded. If more than one obstacle is present, attention must be paid to the fact that the safety areas of the individual obstacles do not overlap. If this cannot be avoided, a common security zone must cover the corresponding obstacles. Section 2.6 shows an applicable solution.

By the determination of the gradients, only the gradient lines of the obstacle nearest to the vehicle are used. This leads to the fact that by driving through obstacles it is switched to the current in each case gradient line field. In order to obtain a smooth transition between two gradient fields it is necessary to use continuous switching instead of binary switching. In Guldner (1997) a range was defined along the boundary line of two neighbouring gradient line fields $\left(d_{\text {line } \max }\right)$, into which the gradients of the individual fields overlap by means of a weighting factor. This priority factor is selected proportional to the distance between vehicle and the boundary line.

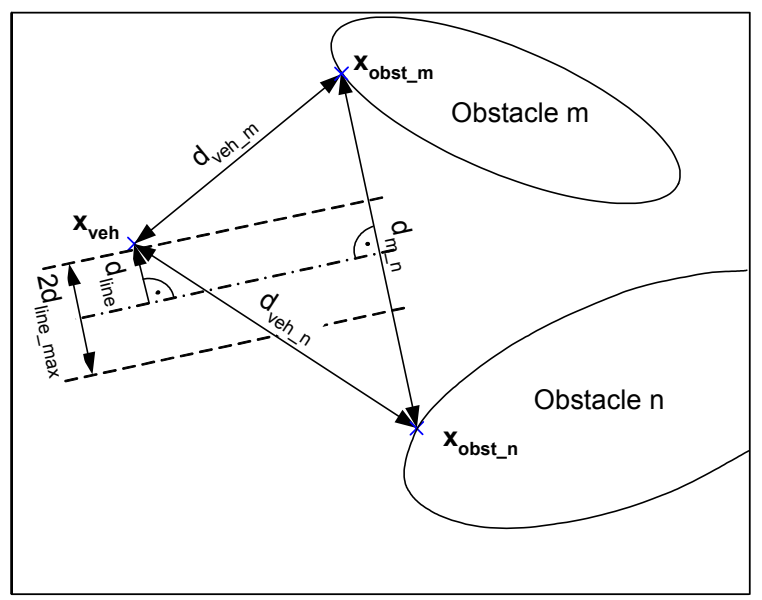

Fig.7. Simplified determination of $\mathrm{d}_{\text {line }}$

A simplified determination of this distance is done via the following equation based on Fig.7.

$$
d_{\text {line }}=\frac{d_{v e h_{-} n}^{2}-d_{v e h_{-} m}^{2}}{2 d_{m_{-} n}}
$$

$d_{v e h \_n}$ and $d_{v e h \_m}$ describe the shortest distance from the current vehicle position to the respective obstacle. The resulting gradient can be expressed as follows:

$$
\mathbf{G}= \begin{cases}\mathbf{G}_{\text {obst_m }} \quad \text { for } d_{\text {line }}>d_{\text {line_max }} \\ \mathbf{G}_{\text {obst_n }} \quad \text { for } d_{\text {line }}<-d_{\text {line_max }} \\ \alpha \mathbf{G}_{\text {obst_m }}+(1-\alpha) \mathbf{G}_{\text {obst } n} \text { for }\left|d_{\text {line }}\right|<d_{\text {line_max }}\end{cases}
$$


with the weighting factor $\alpha$

$$
\alpha=0.5\left(1+\frac{d_{\text {line }}}{d_{\text {line_max }}}\right) .
$$

\subsection{Overlapping safety areas}

If it comes to overlaps between two or more safety zones, a common safety zone must cover these areas. This zone should possess an elliptical form, which includes the individual safety zones completely. However, as little passable area as possible. For the solution of this problem the "computation of smallest ellipsoids around point sets" was applied (Gärtner, 1998). In addition, around each safety zone a polygon was defined, which includes the obstacle. Around the corners of the polygons formed in this way an ellipse is computed using the GCAL library (CGAL, 2004). The computation of the polygon vertices is done on the basis of standardized vertices around a unit circle. These are adapted by scaling and rotation of the appropriate ellipse (see equations (13) and (14)). The vertices $\mathbf{S}_{\mathbf{0}} \ldots \mathbf{n - 1}$ are computed for the first quadrant of the unit circle by computation of the crossing points using equation (19) and the rays defined in equation (18) and adapted for the other quadrants by appropriate sign change (see Fig. 8).

$$
\begin{gathered}
\mathbf{P}_{\mathbf{n}}=\left[\begin{array}{c}
\cos (\mathrm{n} \cdot \alpha) \\
\sin (\mathrm{n} \cdot \alpha)
\end{array}\right] \quad \mathbf{d}_{\mathbf{n}}=\left[\begin{array}{c}
-\sin (\mathrm{n} \cdot \alpha) \\
\cos (\mathrm{n} \cdot \alpha)
\end{array}\right] \\
\mathbf{E}=\mathbf{P}_{\mathbf{n}+\mathbf{1}}-\mathbf{P}_{\mathbf{n}} \quad \mathrm{t}=\frac{\mathbf{E} \times \mathbf{d}_{\mathrm{n}+1}}{\mathbf{d}_{\mathbf{n}} \times \mathbf{d}_{\mathbf{n}+1}} \\
\mathbf{S}_{\mathbf{n}}=\mathbf{P}_{\mathbf{n}}+\mathrm{t} \cdot \mathbf{d}_{\mathbf{n}}
\end{gathered}
$$

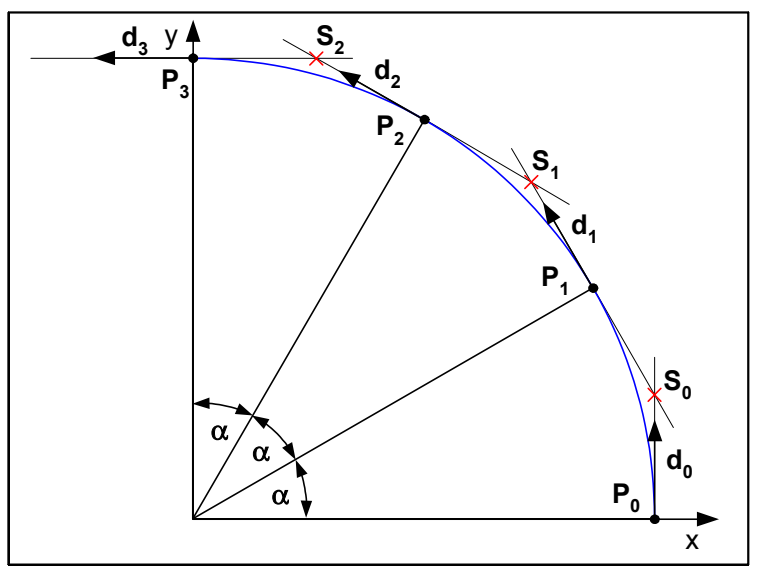

Fig. 8. Determination of the vertices of the unit circle

Fig. 9 shows the determined vertices of the individual safety zones and the ellipse computed from them. In the enlargement it can be seen clearly that the polygon edges tangents the safety zone and does not cut them.

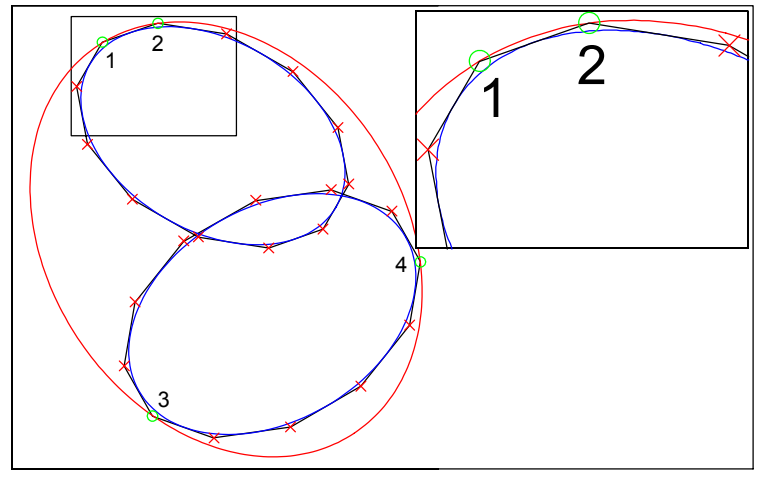

Fig. 9. Smallest enclosing ellipse

The gradient computation inside the ellipse is done in analogy to the method described in section 2.5. If the vehicle position is inside a safety zone the next position $\left(\mathbf{x}_{\mathbf{o b s t} \_} \mathbf{m}\right.$ or $\left.\mathbf{x}_{\mathbf{o b s t} \_\mathbf{n}}\right)$ to the vehicle, the vehicle position $\mathbf{x}_{\mathbf{v e h}}$ is defined. Fig. 10 shows the gradient field formed this way.

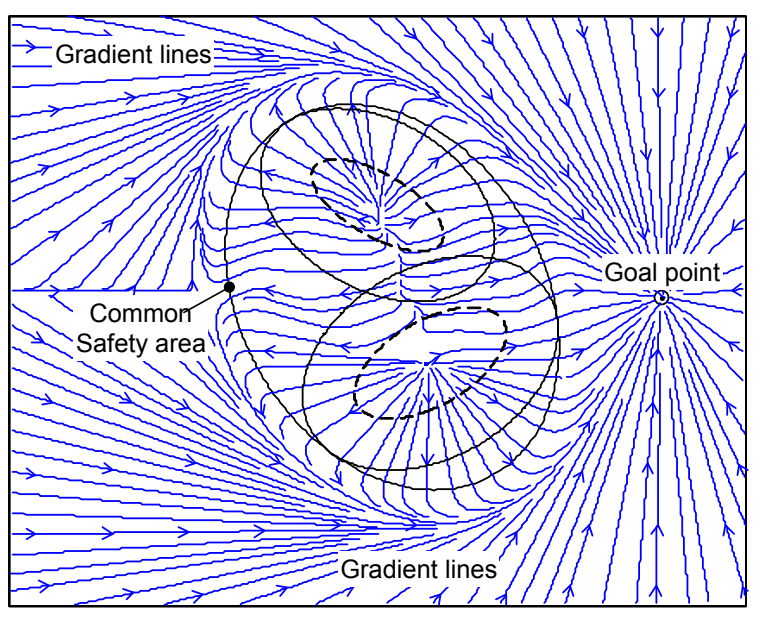

Fig. 10. Gradient lines by common safety areas

\section{TEST RESULTS}

Finally the procedure of the geometrical construction has been compared with the procedure of the harmonious dipole potentials. Therefore an obstacle scenario illustrated in Fig. 11 was defined. The vehicle is to be driven with a cruising speed of $2 \mathrm{~m} / \mathrm{s}$ through the obstacles. It could be shown that with the use of the geometrical construction method the target course change rate $r$ possesses substantially smaller maximum values than by using the procedure of the harmonious dipole potentials (see Fig. 12). This is an important criterion for real applications, since the applied vehicle cannot follow reference trajectory changes very fast, which causes deviations in the gradient lines and thus to possible collisions. The necessary path length for driving through the obstacle scenario with the geometrical procedure amounts up to $9 \%$ less than that with the method of the harmonious dipole potentials. 


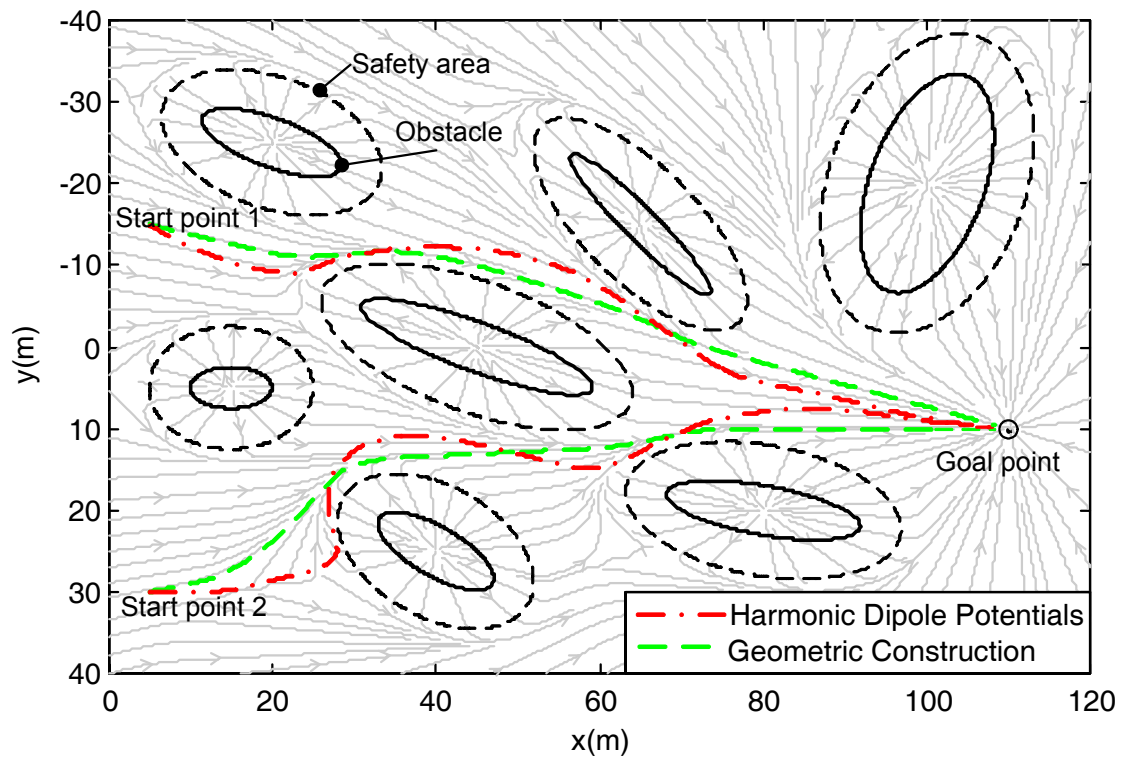

Fig. 11. Paths through the obstacle scenario
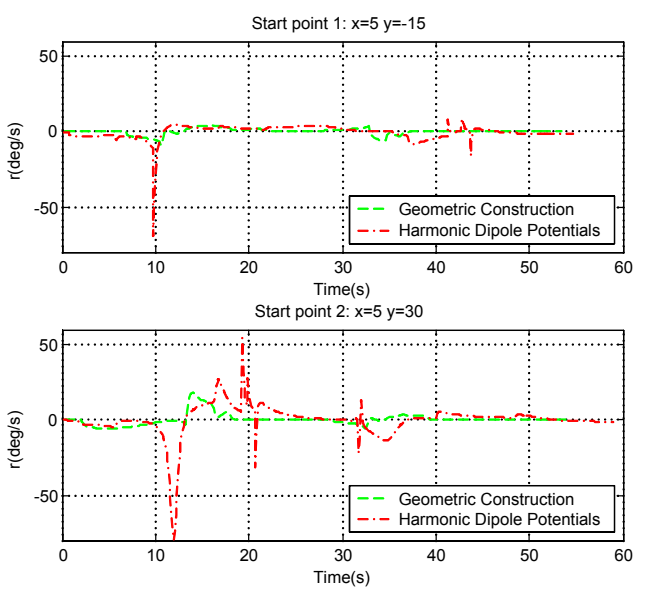

Fig. 12. Evaluation of the reference turning rate $r$

\section{CONCLUSIONS}

In this article a reactive system for the guidance of an underwater vehicle in obstacle avoidance situations was presented. This system is the local level of the obstacle avoidance system how was developed for the AUV DeepC. The special characteristics of the vehicle (existing arithmetic performance and sensor technology, driving characteristic and manoeuvrability) formed the main criteria for the selection and development of the algorithms.

The presented method for the geometrical construction of gradient lines connects the advantages of the method of the artificial harmonious dipole potentials with the demands on optimal path and control driving fashion. The sea-current as well as possible movements of the obstacles were considered in a simple manner by the construction of the gradient lines by formation of a substitute form for the obstacle.

The implementation of the presented algorithms on the goal platform and the tests with the AUV simulator has been finished in summer 2004. The first free water attempts with the AUV will take place in spring 2005.

\section{ACKNOWLEDGMENT}

This work is part of the current research project "DeepC", which is being promoted by the BMBF. (Promotion no: 03SX104E). I would like to thank Professor J. Wernstedt, who made it possible for me to work in this interesting project, my colleague Divas Karimanzira for good co-operation and assistance during the project as well as Uwe Möller and Hendrik Schelenz from ATLAS ELEKTRONIK GmbH Bremen for the good teamwork.

\section{REFERENCES}

BMBF (2001). Förderprojekt des BMBF: DeepC. Aktiv autonomes Unterwasserfahrzeug für große Tauchtiefen. Förderkennzeichen: 03SX104E.

CGAL (2004). http://www.cgal.org/

DeepC (2004). http://www.deepc-auv.de/deepc/ DeepC.htm

Eichhorn, M. (2004). An Obstacle Avoidance System for an Autonomous Underwater Vehicle. in Proceedings of the International Symposium on Underwater Technology, Taipei, Taiwan, April 20-23 2004.

Gärtner, B. and Schönherr S. (1998) Smallest Enclosing Ellipses An Exact and Generic Implementation in $\mathrm{C}++$ Report B 98-05, Freie Universität Berlin, Institut für Informatik. http://www.inf.fu-berlin.de/inst/ubs/r-b-98-05. abstract.html

Guldner, J. (1995). Intelligentes hierarchisches Regelungskonzept für autonome mobile Robotersysteme. Düsseldorf, Germany: VDIVerlag.

Guldner, J. (1997). Lokale Kollisionsvermeidung für mobile Roboter mittels künstlicher harmonischer Dipol-Potentiale. at Automatisierungstechnik, Volume 45, Issue 01, pp. 24-35. 